\section{Pensar en la producción de conocimientos y reflexionar sobre el campo de la economía social. La contribución del Centro de Estudios de Sociología del Trabajo en la Universidad de Buenos Aires}

\section{Mirta Vuotto}

Docente investigadora de la Universidad de Buenos Aires (UBA), Argentina Directora del Centro de Estudios de Sociología del Trabajo - Facultad de Ciencias Económicas, UBA.
Economía Social y Solidaria /

Desafíos de gestión

RECEPCIÓN: 18/06/15

ACEPTACIÓN FINAL: 27/07/15

\section{Resumen}

La reflexión en torno a la producción científica de un ámbito de investigación es una ocasión propicia para plantear interrogantes sobre su eficacia y reflexionar sobre su contribución al reconocimiento de un campo específico de conocimiento. El artículo refiere al Centro de Estudios de Sociología del Trabajo en la Facultad de Ciencias Económicas de la Universidad de Buenos Aires como ámbito de investigación, para destacar que desde su creación, en 1994, se ha interesado en contribuir al reconocimiento del campo de la economía social.

El texto está estructurado en dos secciones. Luego de introducir algunas referencias sobre los objetivos y actividades del Centro, se caracterizan las investigaciones orientadas al conocimiento de la economía social y se presentan los instrumentos de difusión que dan visibilidad a los resultados de la investigación. A través de estos aspectos se plantea, a modo de conclusión, la especificidad de un campo de estudios interrogándose sobre la aplicabilidad de los conocimientos que surgen de la investigación.

Palabras clave

- investigación

- economía social

- cooperativas

- aplicabilidad de conocimientos

\section{Resumo}

A reflexão acerca da produção científica de um campo de pesquisa constitui uma ocasião propícia para levantar questões sobre a sua eficácia e refletir sobre a sua contribuição para o reconhecimento de uma área específica do conhecimento. $O$ artigo refere-se ao Centro de Estudios de Sociología do Trabalho na Faculdade de Ciências Econômicas de la Universidade de Buenos Aires como um espaço de pesquisa, destacando que desde a sua criação em 1994, interessou-se por contribuir para o reconhecimento do campo da economia social.

O texto está dividido em duas seções. Após a introdução de algumas referências sobre os objetivos e atividades do Centro, se indicam os resultados das pesquisas orientadas ao conhecimento sobre a economia social e, em seguida, se apresentam as ferramentas de divulgação que dão visibilidade aos resultados desses estudos. A partir desses elementos, surge, como conclusão, a especificidade de um campo de estudos, questionando-se sobre a aplicabilidade dos conhecimentos resultantes da investigação.

Palavras-chave

- investigação

- economia social

- cooperativas

- aplicabilidade dos conhecimentos
Para citación de este artículo Vuotto, M. (2015). Pensar en la producción de conocimientos y reflexionar sobre el campo de la economía social. La contribución del Centro de Estudios de Sociología del Trabajo en la Universidad de Buenos Aires. En Revista +E versión digital, (5), pp. 114-119. Santa Fe, Argentina: Ediciones UNL. 


\section{centro tiene como objetivo desarrollar investigaciones sobre la temática laboral focalizando en las organizaciones de la economía social o Tercer Sector y dedicando especial atención a las entidades cooperativas}

\section{Introducción}

El Centro de Estudios de Sociología del Trabajo fue creado en 1994 en el marco de la Secretaría de Investigación de la Facultad de Ciencias Económicas de la Universidad de Buenos Aires. ${ }^{1}$ Está integrado por un grupo interdisciplinario de profesionales que desarrollan actividades de investigación, docencia y extensión vinculadas con la temática del trabajo en las organizaciones. Los miembros del equipo son profesionales formados en diversas disciplinas como sociología, administración, economía y contabilidad, especializados en sociología de las organizaciones, metodología de la investigación social y economía social. También integra este equipo de trabajo un grupo de becarios, tesistas de posgrado y pasantes de investigación.

Específicamente, el Centro tiene como objetivo desarrollar investigaciones sobre la temática laboral focalizando en las organizaciones de la economía social o Tercer Sector y dedicando especial atención a las entidades cooperativas. Se propone, además, difundir resultados e impulsar actividades de transferencia de investigación y docencia a la comunidad académica, a las organizaciones públicas y a la comunidad y generar vínculos de trabajo con instituciones y organizaciones académicas y de la economía social en el ámbito nacional, regional e internacional.

\section{Las investigaciones realizadas} en el campo de la economía social

Algunos de los trabajos desarrollados en el curso de dos décadas permitieron proveer insumos para decisores institucionales del ámbito público. Cabe mencionar al respecto los estudios realizados en forma conjunta con las Facultades de Ciencias Económicas de las Universidades Nacionales de Córdoba, Comahue, Tucumán

1) El Centro depende del Instituto de Investigaciones en Administración, Contabilidad y Métodos Cuantitativos para la Gestión de la Universidad de Buenos Aires. y Cuyo, que se orientaron a caracterizar a las instituciones intermediarias de oferta y demanda de empleo y a identificar la diversidad de estrategias empresariales de búsqueda y selección de personal. Los estudios, de alcance nacional, brindaron resultados al Ministerio de Trabajo para la organización de un programa de oficinas de empleo regionales.

También se pueden destacar los referidos a la temática del sector no lucrativo argentino realizados para el Centro Nacional de Organizaciones de la Comunidad y la participación en las actividades de la Comisión Mixta sobre Criterios de Medición y Construcción de Indicadores del Tercer Sector y en la conformación de la Red de Empresas Sociales. En este ámbito institucional se hizo un relevamiento sobre las instituciones académicas y las investigaciones sobre la temática del sector no lucrativo en la Argentina.

Asimismo, con motivo del Congreso Argentino de las Cooperativas, organizado por la Confederación Cooperativa de la República Argentina en 2012, se elaboraron documentos referenciales relacionados con la formación universitaria en cooperativismo y economía social y la investigación en cooperativismo en instituciones universitarias en la Argentina.

Un segundo grupo de investigaciones se orientó al conocimiento de problemáticas específicas de las organizaciones de la economía social y la transferencia de herramientas de gestión. En este caso algunas fueron realizadas por el equipo del Centro y otras por becarios de posgrado. Las mismas focalizaron los temas de gestión en el campo del cooperativismo de trabajo, de consumo, de vivienda y de crédito, las políticas públicas y los instrumentos de política orientados a la economía social y los procesos de integración en entidades cooperativas.

El interés de este tipo de estudios radica en que la información obtenida fue utilizada por las organizaciones en las que se efectuó la investigación para identificar áreas y sectores problemáticos y sirvió de guía para el desarrollo de sus estrategias empresariales y para la evaluación del alcance de algunas metas. 

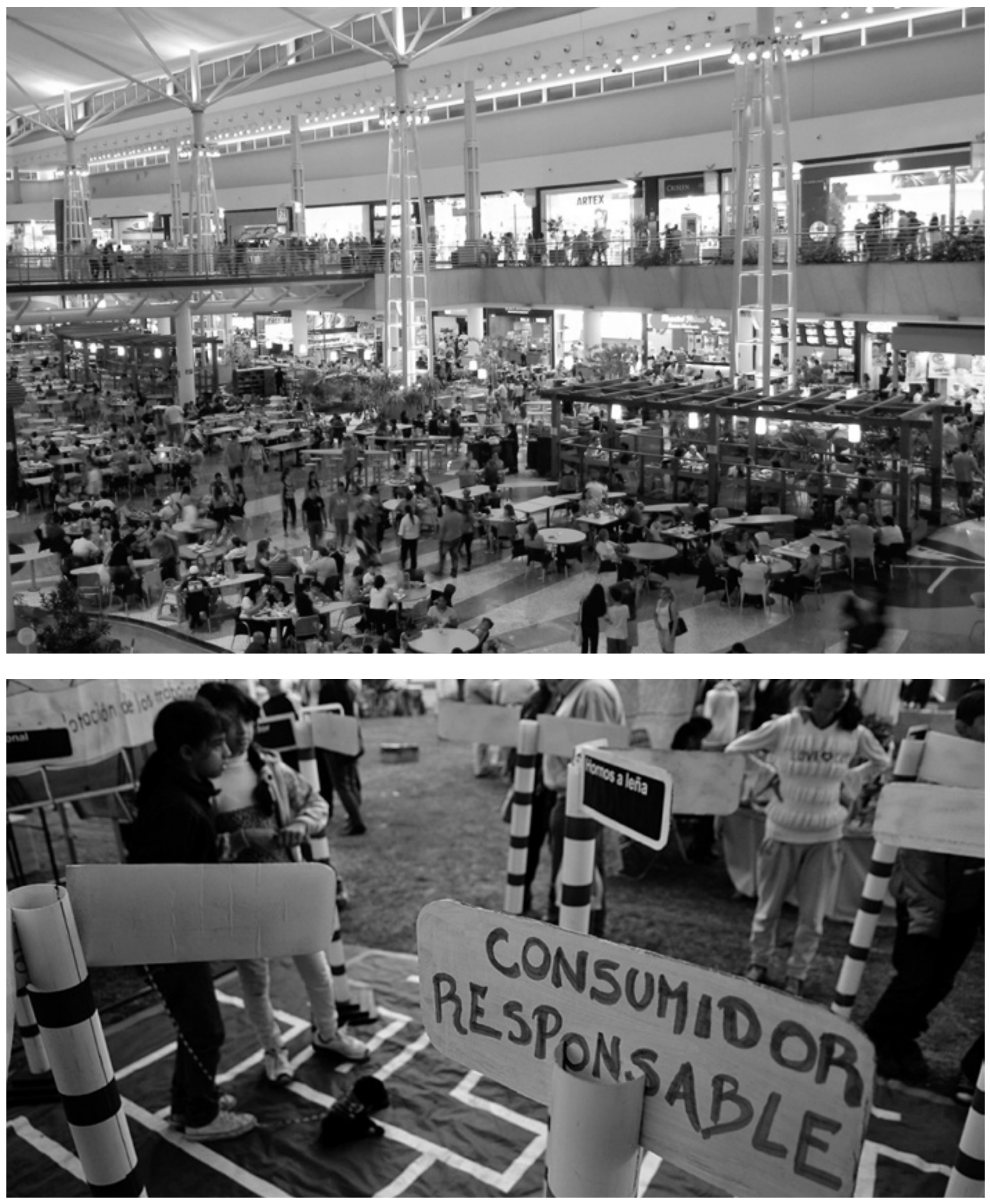
Las investigaciones brindaron además, información que posibilitó a las entidades desarrollar vínculos diversos con el objeto de dar respuestas efectivas a los intereses de sus miembros y ante cambiantes demandas. Esta producción y los resultados obtenidos resultaron también de utilidad para las organizaciones cooperativas de segundo grado.

En esta línea de investigación, el Centro está llevando a cabo un proyecto relativo al surgimiento y desarrollo de las cooperativas en Argentina y Cuba ante las nuevas políticas estatales orientadas al cooperativismo. El estudio comparativo sobre los estilos de gestión de las cooperativas y los aportes al desarrollo local en el último quinquenio está auspiciado por el Ministerio de Ciencia, Tecnología e Innovación Productiva de la República Argentina en el marco de la implementación del Programa de Cooperación Científico Tecnológico con el Ministerio de Ciencia, Tecnología y Medio Ambiente de la República de Cuba.

En conjunto con las anteriores, un tercer grupo de investigaciones se vinculó con el trabajo docente orientándose al análisis de problemáticas organizacionales de la economía social. Por un lado, en el ámbito de la formación de graduados y estudiantes y en la dirección de trabajos de becarios de iniciación en la investigación y de tesistas de maestría y doctorado. Por otro, en la realización de estudios destinados a recoger la opinión de los estudiantes en temas vinculados a la economía social. Específicamente, se indagó en este ámbito por el conocimiento sobre la economía social y sobre el interés de los estudiantes por trabajar en organizaciones cooperativas. Estos trabajos se hicieron con la finalidad de complementar las actividades docentes y estimular capacidades de investigación entre los estudiantes.

De los proyectos de investigación en las cátedras se deben destacar los resultados de un estudio de opinión sobre la imagen del trabajo en las empresas. Este estudio implicó la aplicación de una encuesta a una población de más de 490 estudiantes de la Facultad de Ciencias Económicas de la Universidad de Buenos Aires, y sus resultados, específicamente los referidos al trabajo en las empresas cooperativas, formaron parte del libro Los jóvenes reinventan las cooperativas. Perspectivas de los jóvenes sobre el movimiento cooperativo internacional, coeditado por la Universidad de Victoria en Canadá y la Organización Internacional del Trabajo. Este tipo de estudios ha podido brindar información útil al área académica para el diseño de estrategias sobre los componentes curriculares, en afinidad con los requerimientos de las organizaciones de economía social. Al vincular a través de esos trabajos los contenidos teóricos relativos a la estructura y funcionamiento de distintos tipos de organizaciones con sus referentes institucionales se da por supuesto que los resultados obtenidos favorecen el proceso de aprendizaje y que la experiencia concreta de recolección de la información y su posterior análisis posibilitan una sensibilización frente a los problemas que también plantea la inserción laboral de los futuros graduados.
Y al considerar la naturaleza social de la investigación, es pertinente destacar la importancia del trabajo en redes con grupos en la Argentina y en el exterior.

Entre las actividades de mayor importancia en el país se señala la participación en una red de especialistas convocada por el Ministerio de Educación de la Nación para colaborar con el diseño de los contenidos curriculares de una Tecnicatura en Economía Social que se implementó en el año 2005 y la elaboración de los materiales destinados a los docentes responsables del dictado de la materia Economía Social.

Asimismo, la participación en un grupo de trabajo convocado por la Agenzia di Coordinamento Territoriale Italia-Argentina y la Universidad Nacional de San Martín dio lugar a la elaboración de una propuesta de Ley sobre "Empresa Social y Emprendimientos Sociales de la Provincia de Buenos Aires" que fue presentada en 2010 ante las autoridades de la Cámara de Diputados de la Provincia de Buenos Aires.

Entre las actividades conjuntas con universidades extranjeras y el Comité de Investigación de la Alianza Cooperativa Internacional, el Centro ha sido miembro fundador, en el año 2000, de la Red Latinoamericana de Investigadores en Cooperativismo y continúa participando en la organización de los Encuentros Latinoamericanos de Investigación sobre Cooperativas. Dichos encuentros constituyen el evento científico multidisciplinario regional más significativo en el campo de la investigación en este tema. A través de los mismos se estimula la reflexión sobre las problemáticas del cooperativismo en la región; se promueve la generación de un debate crítico sobre el quehacer cooperativo; se establecen vínculos de colaboración para la investigación y se incentiva el fortalecimiento de relaciones entre el ámbito cooperativo y el ámbito académico.

Los ocho encuentros realizados han favorecido un trabajo colectivo que, de distintas maneras, ayudó a reforzar la legitimidad de la investigación en cooperativismo y propició un mayor reconocimiento de este campo de investigación.

\section{Las actividades de extensión}

Entre las actividades de extensión cabe destacar el desarrollo de tres proyectos: el primero, orientado a fortalecer la gestión de cooperativas de trabajo en la Argentina, está relacionado con una iniciativa de desarrollo de Canadá en la Argentina y el Fondo de transferencia de tecnología Canadá-Cono Sur; el segundo tuvo como objetivo caracterizar la significación del empleo en una cooperativa, basándose en indicadores relativos a la percepción de empleados y directivos sobre su organización y el grado de satisfacción con su trabajo. El tercero, actualmente en desarrollo, consiste en diseñar una red virtual de experiencias cooperativas que facilitará las interacciones e intercambios entre las cooperativas de todos los sectores de actividad, sus usuarios 
y los stakeholders, vinculándolos con las universidades y la comunidad local, proveyendo conocimientos que permitan valorizar su patrimonio económico, social y cultural.

El primer proyecto fue implementado por un equipo del Centro y ejecutado en asociación con 25 cooperativas de trabajo localizadas en el área de la ciudad de Buenos Aires y la provincia de Buenos Aires. Entre sus finalidades se destacan la difusión de conocimientos, de tecnologías y de saber-hacer canadiense a través de actividades de formación e intercambios técnicos y servicios en los cuales las instituciones canadienses poseen competencias que se corresponden con las necesidades y prioridades de sus contrapartes sudamericanas. El programa de formación fue desarrollado por consultores canadienses y se dirigió a los representantes de las cooperativas participantes y al equipo que acompañaría el desarrollo del proyecto en cada organización. Se propuso, a través de la capacitación, lograr un lenguaje común entre los actores involucrados en el proyecto a fin de potenciar el trabajo en equipo y preparar las actividades de acompañamiento. Se abordaron temáticas relativas a la gestión administrativa (gestión de personas, contabilidad y finanzas, comercialización, formulación y evaluación de proyectos, etc.) y la gestión cooperativa (gobierno y representación). El programa de acompañamiento, con base en las demandas efectuadas por las cooperativas a través de sus representantes y los resultados de un estudio diagnóstico previo, permitió estructurar una serie de acciones con el objetivo de facilitar la transferencia de tecnología para atender las demandas realizadas. Los acompañantes tuvieron un papel relevante en el monitoreo de los resultados del trabajo en las cooperativas y en el aporte a la resolución de las problemáticas planteadas. Para lograr modalidades más amplias de concertación entre las organizaciones y entre éstas y las instituciones relacionadas con el sector cooperativo y de la economía social fue diseñado un portal electrónico del proyecto tratando de promover una modalidad de vinculaciones mutuas, a fin de compartir experiencias y potenciar demandas, capacitando a los actores en la utilización del sistema. Mientras que el desarrollo de las actividades relativas a la formación fue propuesto por la ONG Alternatives, impulsora del proyecto, basándose en las informaciones sobre las cooperativas participantes, las tareas de acompañamiento técnico surgieron del ajuste entre los requerimientos y necesidades de las cooperativas y los recursos del proyecto. El esquema de intervención planteado por el equipo y la discusión de las actividades a desarrollar en las cooperativas, luego de la etapa de capacitación, posibilitó consensuar las principales líneas de trabajo, identificando en cada caso las fortalezas y debilidades referidas a la estructura de la organización y procesos de trabajo, la naturaleza de las prácticas laborales y asociativas y las principales necesidades y demandas. Como síntesis de las contribuciones del proyecto se elaboró y publicó el libro Guía de consulta para la gestión de cooperativas de trabajo. ${ }^{2}$ El material es una herramienta que complementa el trabajo llevado a cabo durante las actividades de capacitación y acompañamiento efectuadas con las cooperativas participantes en el proyecto y contiene informaciones que permiten una mayor comprensión sobre la organización cooperativa de trabajo y su funcionamiento. El segundo proyecto se abocó al estudio de las dimensiones objetivas y subjetivas de la calidad del empleo en una entidad cooperativa de segundo grado. Los ámbitos incluidos en el estudio fueron la calidad del trabajo y del empleo, el empoderamiento y el grado de autonomización de sus empleados y directivos. Esos ámbitos fueron analizados contemplando las siguientes dimensiones: organización del trabajo, salario y sistemas de remuneración, seguridad y flexibilidad, actitudes y desarrollo, compromiso y representación por parte de los empleados. Los resultados obtenidos en el estudio permitieron caracterizar la imagen que tiene el trabajador sobre su cooperativa —en lo relativo a su empleo-y su grado de implicación con la organización, posibilitando una mejor comprensión acerca de la forma en que el trabajo impacta en la calidad de la vida laboral.

Por último, el proyecto de promoción de una Ruta Argentina de Cooperativas, que se realiza en colaboración con la Universidad Nacional de Cuyo y la Universidad Católica Argentina con la participación de instituciones cooperativas consiste en visibilizar un conjunto de experiencias cooperativas, con un fuerte énfasis en la valorización de los recursos locales, la herencia territorial y la identidad cooperativa para crear una plataforma virtual que tenga un impacto positivo en las organizaciones incluidas en la ruta y en sus comunidades.

\section{Un espacio de información para dar visibilidad a los resultados de la investigación}

Para difundir las actividades de investigación realizadas en torno a la temática sociorganizacional y laboral se edita anualmente una revista científica y documentos de trabajo de publicación trimestral: 
- La Revista del Centro de Estudios de Sociología del Trabajo es una publicación arbitrada de carácter interdisciplinario en el campo de los estudios socioorganizacionales y laborales. Está orientada a difundir los resultados de estudios teóricos y empíricos y a estimular debates e intercambios entre especialistas de distintas regiones. Los autores de los artículos son argentinos y extranjeros y los artículos se inscriben generalmente en una perspectiva de investigación. Se dirige a investigadores y a un público más amplio de profesionales, docentes y estudiantes en disciplinas que integran las Ciencias Sociales. Se publica en edición impresa y electrónica, con acceso abierto en https://ojs.econ.uba.ar/ojs/index.php/CESOT

La Revista está incorporada en el Núcleo Básico de Revistas Científicas Argentinas y se encuentra indizada en las bases de datos: Catálogo Latindex, E-revistas, La Criée: périodiques en ligne y CIDEC. Se publica anualmente en el mes de abril.

- En el marco de su política de producción y difusión el Centro dispone un fondo de documentos constituido por referencias sobre los principales temas de interés. La serie Documentos del Centro de Estudios de Sociología del Trabajo se publica desde 1996. Los documentos contienen resultados de investigaciones realizadas por miembros del Centro, la presentación de actividades académicas y traducciones de artículos publicados en revistas especializadas en economía social.

\section{A modo de conclusión}

En el artículo se han expuesto algunos resultados relativos a la tarea de investigación del Centro de Estudios de Sociología del Trabajo. En conjunto permiten responder al propósito que motivó la creación de este Centro y expresar a través de su labor investigativa que la economía social debe ser objeto de la investigación académica. Sin embargo, es necesario ir más lejos, plantear la necesidad de sensibilizar sobre su aplicabilidad en las propias organizaciones ${ }^{3}$ y mostrar los beneficios que pueden resultar de los resultados de la investigación.

De esto se desprende que la pertinencia y especificidad de este campo de estudios no es solamente una cuestión académica, sino también y en especial una cuestión sociopolítica. El conocimiento va de la mano del reconocimiento y la cientificidad de un área de investigación no es independiente de la legitimidad que las instituciones le confieren.
Los archivos de la revista están disponibles en el sitio de publicaciones del Centro de Estudios de Sociología del Trabajo:

http://home.econ.uba.ar/economicas/ cesot-numeros-publicados Se invita a la publicación de artículos y se indican las instrucciones a los autores y las normas de publicación http://home.econ.uba.ar/economicas/ cesot-normas-depublicacion
Los archivos de los documentos están disponibles en el sitio de publicaciones del Centro de Estudios de Sociología del Trabajo: http://home.econ.uba.ar/economicas/ cesot-documentos
Dadas las implicancias que esto supone para la labor cotidiana, parecería necesario adoptar la aplicabilidad como criterio para orientar nuestras elecciones de trabajo y también para evaluar los resultados de nuestra investigación. Es un buen reaseguro para superar la posición de quien entiende que el investigador no debe convertirse simplemente en espejo o intérprete de la realidad. Así, la doble perspectiva: contemplar la especificidad del campo de estudio y garantizar la aplicabilidad de los resultados de investigación podrán estimular la producción de nuevos puntos de vista y saberes teóricos y prácticos incentivando nuestra capacidad para hacer emerger cuestiones útiles para la acción, valorizables desde el punto de vista de la investigación y con capacidad para producir ventajosas sinergias para la acción conjunta de actores de la economía social e investigadores.
3) Resulta pertinente recordar que los estudios de la organización se beneficiarían mucho de orientarse más hacia los fenómenos y de preocuparse más por la aplicabilidad de sus ideas [Pffefer J. (2000). Nuevos rumbos en la teoría de la organización. Problemas y posibilidades (276). Oxford University]. 